\title{
Impact of finite density on spectroscopic parameters of decuplet baryons
}

\author{
K. Azizi ${ }^{1}$, N. Er ${ }^{2}$, H. Sundu ${ }^{3}$ \\ 1 Department of Physics, Doğuş University, Acıbadem-Kadıköy, 34722 Istanbul, Turkey \\ 2 Department of Physics, Abant İzet Baysal University, Gölköy Kampüsü, 14980 Bolu, Turkey \\ 3 Department of Physics, Kocaeli University, 41380 Izmit, Turkey
}

( $\Omega$ Dated: October 9, 2018)

\begin{abstract}
The decuplet baryons, $\Delta, \Sigma^{*}, \Xi^{*}$ and $\Omega^{-}$, are studied in nuclear matter by using the in-medium QCD sum rules. By fixing the three momentum of the particles under consideration at the rest frame of the medium, the negative energy contributions are removed. It is obtained that the parameters of the $\Delta$ baryon are more affected by the medium against the $\Omega^{-}$state, containing three strange quarks, whose mass and residue do not affected by the medium, considerably. We also find the vector and scalar self energies of these baryons in nuclear matter. By the recent progresses at $\bar{P} A N D A$ experiment at FAIR and NICA facility it may be possible to study the in-medium properties of such states even the multi-strange $\Xi^{*}$ and $\Omega^{-}$systems in near future.

PACS numbers: 21.65.-f, 14.20.-c, 14.20.Dh, 14.20.Jn, 11.55.Hx
\end{abstract}

\section{INTRODUCTION}

The investigations of the properties of hadrons under extreme conditions have been in the focus of much attention for many years. Such investigations are very important in the study of the internal structure of the dense astrophysical objects like neutron stars. The formation of neutron stars is influenced by all four known fundamental interactions. Hence, understanding of their nature can help us in the course of unification of all fundamental forces within a common theoretical framework, which is one of the biggest challenges for physics. The recent observation of massive neutron stars with roughly twice the solar mass [1, 2] has stimulated the focuses on the equation of state of the dense nuclear matter (see for instance $[3-6]$ ). However, the expected appearance of hyperons at about two times nuclear density, called "hyperon puzzle" remains an unresolved mystery in neutron stars (concerning the appearance of hyperons in neutron stars see for example [7, 8] ). It has also found that $\Delta$ isobars appear at a density of the order of $2 \div 3$ times nuclear matter saturation density, and a " $\Delta$ puzzle" exists, similar to the "hyperon puzzle" if the potential of the $\Delta$ in nuclear matter is close to the one indicated by the experimental data 9]. More theoretical and experimental investigations on the properties of strange and non-strange light baryons in dense medium are needed to solve such puzzles.

From the experimental side, the bound nuclear systems with one, two or three units of strangeness are poorly known compared to that of the non-strange states like nucleons. The large production probability of various hyperon-antihyperon pairs in antiproton collisions will provide opportunities for series of new studies on the behavior of the systems containing two or even more units of strangeness at the $\bar{P}$ ANDA experiment at FAIR. By the progresses made, it will be possible to study the inmedium properties of the doubly strange $\Lambda \Lambda$-hypernuclei as well as the multi-strange $\Xi^{-}, \bar{\Xi}^{+}$and $\Omega^{-}$systems in near future [10].

From the theoretical side, the effects of nuclear medium on the physical parameters of the nucleon have been widely investigated in the literature (see for instance [1115. and references therein). But, we have only a few studies dedicated to the in-medium properties of hyperons and decuplet baryons in the literature (for instance see [16 22]). In the present study, we investigate the impact of nuclear matter on some spectroscopic parameters of the $\Delta, \Sigma^{*}, \Xi^{*}$ and $\Omega^{-}$decuplet baryons. In particular, we calculate the mass and residue as well as the scalar and vector self energies of these baryons using the well established in-medium QCD sum rule approach. We compare the in-medium results with those obtained at $\rho=0$ or vacuum and find the corresponding shifts. To remove the contributions of the negative energy particles, we work at the rest frame of the nuclear matter and fix the three momentum of the particles under consideration.

\section{II. $\Delta, \Sigma^{*}, \Xi^{*}$ AND $\Omega^{-}$BARYONS IN NUCLEAR MATTER}

In this section we aim to construct sum rules for the mass, residue and vector self energy of the decuplet baryons and numerically analyze the obtained results. To this end and in accordance with the general philosophy of the QCD sum rule approach,we start with a correlation function as the building block of the method:

$$
\Pi_{\mu \nu}(p)=i \int d^{4} x e^{i p \cdot x}\left\langle\psi_{0}\left|T\left[\eta_{\mu, D}(x) \bar{\eta}_{\nu, D}(0)\right]\right| \psi_{0}\right\rangle,
$$

where $p$ is the four momentum of the decuplet $(D)$ baryon, $\left|\psi_{0}\right\rangle$ is the ground state of the nuclear matter and $\eta_{\mu, D}$ is the interpolating current of the $D$ baryon. The general form of the interpolating current for decu- 


\begin{tabular}{|c|c|c|c|c|}
\hline \hline & $A_{D}$ & $q_{1}$ & $q_{2}$ & $q_{3}$ \\
\hline$\Sigma^{*}$ & $\sqrt{2 / 3}$ & $\mathrm{u}$ & $\mathrm{d}$ & $\mathrm{s}$ \\
$\Delta^{0}$ & $\sqrt{1 / 3}$ & $\mathrm{~d}$ & $\mathrm{~d}$ & $\mathrm{u}$ \\
$\Xi^{*}$ & $\sqrt{1 / 3}$ & $\mathrm{~s}$ & $\mathrm{~s}$ & $\mathrm{u}$ \\
$\Omega^{-}$ & $1 / 3$ & $\mathrm{~s}$ & $\mathrm{~s}$ & $\mathrm{~s}$ \\
\hline \hline
\end{tabular}

TABLE I: The value of the normalization constant $A_{D}$ and the quark flavors $q_{1}, q_{2}, q_{3}$ for the decuplet baryons.

plet baryons in a compact form reads ,

$$
\begin{aligned}
\eta_{\mu, D} & =A_{D} \epsilon^{a b c}\left\{\left(q_{1}^{a T} C \gamma_{\mu} q_{2}^{b}\right) q_{3}^{c}+\left(q_{2}^{a T} C \gamma_{\mu} q_{3}^{b}\right) q_{1}^{c}\right. \\
& \left.+\left(q_{3}^{a T} C \gamma_{\mu} q_{1}^{b}\right) q_{2}^{c}\right\}
\end{aligned}
$$

where $a, b, c$ are color indices, $C$ is the charge conjugation operator and $A_{D}$ is the normalization constant. The quark content and value of $A_{D}$ for different members are given in table I 23]. We will calculate the aforementioned correlation function in two representations: hadronic and OPE (operator product expansion). By equating these two representations, one can get the QCD sum rules for the aimed physical quantities.

\section{A. Hadronic Representation}

The correlation function in the hadronic side is obtained by inserting a complete set of baryonic state with the same quantum numbers as the interpolating current. After performing the integral over four- $x$, we get

$$
\begin{aligned}
\Pi_{\mu \nu}^{\text {Had }}(p) & =-\frac{\left\langle\psi_{0}\left|\eta_{\mu, D}(0)\right| D\left(p^{*}, s\right)\right\rangle\left\langle D\left(p^{*}, s\right)\left|\bar{\eta}_{\nu, D}(0)\right| \psi_{0}\right\rangle}{p^{* 2}-m_{D}^{* 2}} \\
& +\ldots,
\end{aligned}
$$

where $\left|D\left(p^{*}, s\right)\right\rangle$ is the decuplet baryon state with spin $s$ and in-medium four momentum $p^{*}, m_{D}^{*}$ is the modified mass of the decuplet baryon in medium and ... indicates the contributions of the higher states and continuum. The matrix elements in Eq. (3) can be represented as

$$
\begin{aligned}
& \left\langle\psi_{0}\left|\eta_{\mu, D}(0)\right| D\left(p^{*}, s\right)\right\rangle=\lambda_{D}^{*} u_{\mu}\left(p^{*}, s\right), \\
& \left\langle D\left(p^{*}, s\right)\left|\bar{\eta}_{\nu, D}(0)\right| \psi_{0}\right\rangle=\bar{\lambda}_{D}^{*} \bar{u}_{\nu}\left(p^{*}, s\right),
\end{aligned}
$$

where $u_{\mu}\left(p^{*}, s\right)$ is the in-medium Rarita-Schwinger spinor and $\lambda_{D}^{*}$ is the modified residue or the coupling strength of the decuplet baryon to nuclear medium. Inserting Eq. (44) into Eq. (3) and summing over the spins of the $D$ baryon one can, in principle, find the hadronic side of the correlation function. Before that, it should be remarked that the current $\eta_{\mu, D}$ couples to both the spin- $1 / 2$ octet states and the spin- $3 / 2$ decuplet states. In order to get only the contributions of the decuplet baryons, the contributions of the unwanted spin- $1 / 2$ states must be removed from the correlation function. For this aim, we come next with the following procedure. The matrix element of $\eta_{\mu, D}$ between the spin- $1 / 2$ and in-medium states can be decomposed as

$$
\left\langle\psi_{0}\left|\eta_{\mu, D}(0)\right| \frac{1}{2}\left(p^{*}\right)\right\rangle=\left(C_{1} p_{\mu}^{*}+C_{2} \gamma_{\mu}\right) u\left(p^{*}\right),
$$

where $C_{1}$ and $C_{2}$ are constants and $u\left(p^{*}\right)$ is the inmedium Dirac spinor of momentum $p^{*}$. By multiply both sides of the above equation with $\gamma^{\mu}$ and using the condition $\eta_{\mu, D} \gamma^{\mu}=0$, we immediately find the constant $C_{1}$ in terms of $C_{2}$. Hence,

$$
\left\langle\psi_{0}\left|\eta_{\mu, D}(0)\right| \frac{1}{2}\left(p^{*}\right)\right\rangle=C_{2}\left(-\frac{4}{m_{1 / 2}^{*}} p_{\mu}^{*}+\gamma_{\mu}\right) u\left(p^{*}\right),
$$

where $m_{1 / 2}^{*}$ is the modified mass of the spin- $1 / 2$ baryons. It can be easily seen that the unwanted contributions of the spin- $1 / 2$ states are proportional to $p_{\mu}^{*}$ and $\gamma_{\mu}$. By ordering the Dirac matrices as $\gamma_{\mu} \not \not^{*} \gamma_{\nu}$ and setting to zero the terms with $\gamma_{\mu}$ in the beginning and $\gamma_{\nu}$ at the end and those proportional to $p_{\mu}^{*}$ and $p_{\nu}^{*}$, the contributions from the unwanted spin- $1 / 2$ states can be easily eliminated.

Now, we insert Eq. (4) into Eq. (3) and use the summation over spins of the Rarita-Schwinger spinor as

$$
\begin{aligned}
\sum_{s} u_{\mu}\left(p^{*}, s\right) \bar{u}_{\nu}\left(p^{*}, s\right) & =-\left(\not p^{*}+m_{D}^{*}\right)\left[g_{\mu \nu}-\frac{1}{3} \gamma_{\mu} \gamma_{\nu}\right. \\
& \left.-\frac{2 p_{\mu}^{*} p_{\nu}^{*}}{3 m_{D}^{* 2}}+\frac{p_{\mu}^{*} \gamma_{\nu}-p_{\nu}^{*} \gamma_{\mu}}{3 m_{D}^{*}}\right],
\end{aligned}
$$

as a result of which we get

$$
\begin{aligned}
\Pi_{\mu \nu}^{H a d}(p) & =\frac{\lambda_{D}^{*} \bar{\lambda}_{D}^{*}\left(p^{*}+m_{D}^{*}\right)}{p^{* 2}-m_{D}^{* 2}}\left[g_{\mu \nu}-\frac{1}{3} \gamma_{\mu} \gamma_{\nu}\right. \\
& \left.-\frac{2 p_{\mu}^{*} p_{\nu}^{*}}{3 m_{D}^{* 2}}+\frac{p_{\mu}^{*} \gamma_{\nu}-p_{\nu}^{*} \gamma_{\mu}}{3 m_{D}^{*}}\right]+\ldots
\end{aligned}
$$

To proceed, we would like to mention that the inmedium momentum and the modified mass can be written in terms of the self energies $\Sigma_{\mu, \nu}$ and $\Sigma^{S}$ as $p_{\mu}^{*}=$ $p_{\mu}-\Sigma_{\mu, v}$ and $m_{D}^{*}=m_{D}+\Sigma^{S}$, where $\Sigma^{S}$ is the scalar self energy. The self-energy $\Sigma_{\mu, v}$ can also be written in a general form as

$$
\Sigma_{\mu, v}=\Sigma_{v} u_{\mu}+\Sigma_{v}^{\prime} p_{\mu}
$$

where $\Sigma_{v}$ is called the vector self energy and $u_{\mu}$ is the four velocity of the nuclear medium. In the mean-field approximation, the scalar and vector self energies are obtained to be real and independent of momentum and the $\Sigma_{\nu}^{\prime}$ is taken to be identically zero [11, 24]. In this context, particles of any three-momentum appear as stable quasi-particles with self energies that are roughly linear in the density up to nuclear matter density [11, 25]. We perform the calculations in the rest frame of the nuclear medium, i.e. $u_{\mu}=(1,0)$ and at fixed three-momentum 
of D baryon, $|\vec{p}|$. We get

$$
\begin{aligned}
\Pi_{\mu \nu}^{H a d}\left(p_{0}, \vec{p}\right)= & \lambda_{D}^{*} \bar{\lambda}_{D}^{*} \frac{\left(\not p-\Sigma_{v} \not \mu+m_{D}^{*}\right)}{p^{2}+\Sigma_{v}^{2}-2 p_{0} \Sigma_{v}-m_{D}^{* 2}}\left[g_{\mu \nu}\right. \\
& -\frac{1}{3} \gamma_{\mu} \gamma_{\nu}-\frac{2}{3 m_{D}^{* 2}}\left(p_{\mu} p_{\nu}-\Sigma_{v} p_{\mu} u_{\nu}\right. \\
& \left.-\Sigma_{v} u_{\mu} p_{\nu}+\Sigma_{v}^{2} u_{\mu} u_{\nu}\right)+\frac{1}{3 m_{D}^{*}}\left(p_{\mu} \gamma_{\nu}\right. \\
& \left.\left.-\Sigma_{v} u_{\mu} \gamma_{\nu}-p_{\nu} \gamma_{\mu}+\Sigma_{v} u_{\nu} \gamma_{\mu}\right)\right] \\
& +\ldots,
\end{aligned}
$$

where $p_{0}=p \cdot u$ is the energy of the quasi-particle. After ordering of the Dirac matrices and eliminating the unwanted spin-1/2 contributions, we get

$$
\begin{aligned}
\Pi_{\mu \nu}^{H a d}\left(p_{0}, \vec{p}\right)= & \frac{\lambda_{D}^{*} \bar{\lambda}_{D}^{*}}{\left(p_{0}-E_{p}\right)\left(p_{0}-\bar{E}_{p}\right)}\left[m_{D}^{*} g_{\mu \nu}+g_{\mu \nu} \not p\right. \\
& \left.-\Sigma_{v} g_{\mu \nu} \not \mu\right]+\ldots
\end{aligned}
$$

where , $E_{p}=\Sigma_{v}+\sqrt{|\vec{p}|^{2}+m_{D}^{* 2}}$ and $\bar{E}_{p}=\Sigma_{v}-$ $\sqrt{|\vec{p}|^{2}+m_{D}^{* 2}}$ are the positions of the positive- and negative energy poles, respectively. One can write the above equation as an integral representation in terms of the spectral density,

$$
\Pi_{\mu \nu}^{H a d}\left(p_{0}, \vec{p}\right)=\frac{1}{2 \pi i} \int_{-\infty}^{\infty} d \omega \frac{\Delta \rho_{\mu \nu}^{H a d}\left(p_{0}, \vec{p}\right)}{\omega-p_{0}}
$$

where the spectral density $\Delta \rho_{\mu \nu}^{H a d}\left(p_{0}, \vec{p}\right)$, defining by

$$
\begin{aligned}
& \Delta \rho_{\mu \nu}^{\mathrm{Had}}\left(p_{0}, \vec{p}\right) \\
& =\operatorname{Lim}_{\epsilon \rightarrow 0^{+}}\left[\Pi_{\mu \nu}^{\mathrm{Had}}(\omega+i \epsilon, \vec{p})-\Pi_{\mu \nu}^{H a d}(\omega-i \epsilon, \vec{p})\right]
\end{aligned}
$$

is given as

$$
\begin{aligned}
\Delta \rho_{\mu \nu}^{H a d}\left(p_{0}, \vec{p}\right)= & -\frac{1}{2 \sqrt{m_{D}^{* 2}+|\vec{p}|^{2}}} \lambda_{D}^{*} \bar{\lambda}_{D}^{*}\left[m_{D}^{*} g_{\mu \nu}+g_{\mu \nu} \not p\right. \\
& \left.-\Sigma_{v} g_{\mu \nu} \not \mu\right]\left[\delta\left(\omega-E_{p}\right)-\delta\left(\omega-\bar{E}_{p}\right)\right] .
\end{aligned}
$$

The next step is to exclude the negative-energy pole contribution by multiplying the correlation function with the weight function $\left(\omega-\bar{E}_{p}\right) e^{\frac{-\omega^{2}}{M^{2}}}$ and performing the integral over $\omega$ from $-\omega_{0}$ to $\omega_{0}$, i.e.

$$
\Pi_{\mu \nu}^{H a d}\left(p_{0}, \vec{p}\right)=\int_{-\omega_{0}}^{\omega_{0}} d \omega \Delta \rho_{\mu \nu}^{H a d}(\omega, \vec{p})\left(\omega-\bar{E}_{p}\right) e^{-\frac{\omega^{2}}{M^{2}}}
$$

where $\omega_{0}$ is the threshold parameter and $M^{2}$ is the Borel mass parameter which shall be fixed later. After performing the integral in Eq. (15), the hadronic side of the correlation function takes its final form in terms of the corresponding structures,

$$
\Pi_{\mu \nu}^{\mathrm{Had}}\left(p_{0}, \vec{p}\right)=\lambda_{D}^{* 2} e^{-E_{p}^{2} / M^{2}}\left[m_{D}^{*} g_{\mu \nu}+g_{\mu \nu} \not p-\Sigma_{v} g_{\mu \nu} \not \mu\right] .
$$

\section{B. OPE Representation}

The OPE side of the correlation function is calculated at the large space-like region $p^{2} \ll 0$ in terms of QCD degrees of freedom. One can write the OPE side of the correlation function, in terms of the involved structures, as

$$
\begin{aligned}
\Pi_{\mu \nu}^{O P E}\left(p_{0}, \vec{p}\right) & =\Pi_{1}\left(p_{0}, \vec{p}\right) g_{\mu \nu}+\Pi_{2}\left(p_{0}, \vec{p}\right) \not p g_{\mu \nu} \\
& +\Pi_{3}\left(p_{0}, \vec{p}\right) \not \mu g_{\mu \nu}
\end{aligned}
$$

where the $\Pi_{i}\left(p_{0}, \vec{p}\right)$ functions, with $i=1,2$ or 3 , can be written in terms of the spectral densities $\Delta \rho_{i}\left(p_{0}, \vec{p}\right)$ in OPE side as

$$
\Pi_{i}\left(p_{0}, \vec{p}\right)=\frac{1}{2 \pi i} \int_{-\infty}^{\infty} d w \frac{\Delta \rho_{i}\left(p_{0}, \vec{p}\right)}{w-p_{0}}
$$

where $\Delta \rho_{i}\left(p_{0}, \vec{p}\right)$ are the imaginary parts of $\Pi_{i}\left(p_{0}, \vec{p}\right)$ functions obtaining from the OPE version of Eq. (13). The main aim, in the present subsection, is to find the $\Delta \rho_{i}\left(p_{0}, \vec{p}\right)$ spectral densities, by using of which we can find the $\Pi_{i}\left(p_{0}, \vec{p}\right)$ functions in OPE side. To proceed, we start with the correlation function in Eq. (1). By substituting the explicit form of the interpolating current for the decuplet baryons under consideration into the correlation function in Eq. (1) and after contracting out all the quark pairs using the Wick's theorem, we get 


$$
\begin{aligned}
& \Pi_{\mu \nu}^{O P E, \Delta}(p)=\frac{i}{3} \epsilon_{a b c} \epsilon_{a^{\prime} b^{\prime} c^{\prime}} \int d^{4} x e^{i p x}\left\langle\left\{ 2 S_{d}^{c a^{\prime}}(x) \gamma_{\nu} S_{d}^{\prime a b^{\prime}}(x) \gamma_{\mu} S_{u}^{b c^{\prime}}(x)-2 S_{d}^{c b^{\prime}}(x) \gamma_{\nu} S_{d}^{\prime a a^{\prime}}(x) \gamma_{\mu} S_{u}^{b c^{\prime}}(x)\right.\right. \\
& +4 S_{d}^{c b^{\prime}}(x) \gamma_{\nu} S_{u}^{\prime b a^{\prime}}(x) \gamma_{\mu} S_{d}^{a c^{\prime}}(x)+2 S_{u}^{c a^{\prime}}(x) \gamma_{\nu} S_{d}^{\prime a b^{\prime}}(x) \gamma_{\mu} S_{d}^{b c^{\prime}}(x) \\
& -2 S_{u}^{c a^{\prime}}(x) \gamma_{\nu} S_{d}^{\prime b b^{\prime}}(x) \gamma_{\mu} S_{d}^{a c^{\prime}}(x)-S_{u}^{c c^{\prime}}(x) \operatorname{Tr}\left[S_{d}^{b a^{\prime}}(x) \gamma_{\nu} S_{d}^{\prime a b^{\prime}}(x) \gamma_{\mu}\right] \\
& \left.\left.+S_{u}^{c c^{\prime}}(x) \operatorname{Tr}\left[S_{d}^{b b^{\prime}}(x) \gamma_{\nu} S_{d}^{\prime a a^{\prime}}(x) \gamma_{\mu}\right]-4 S_{d}^{c c^{\prime}}(x) \operatorname{Tr}\left[S_{u}^{b a^{\prime}}(x) \gamma_{\nu} S_{d}^{\prime a b^{\prime}}(x) \gamma_{\mu}\right]\right\}\right\rangle \\
& \Pi_{\mu \nu}^{O P E, \Sigma^{*}}(p)=-\frac{2 i}{3} \epsilon_{a b c} \epsilon_{a^{\prime} b^{\prime} c^{\prime}} \int d^{4} x e^{i p x}\left\langle\left\{ S_{d}^{c a^{\prime}}(x) \gamma_{\nu} S_{u}^{\prime b b^{\prime}}(x) \gamma_{\mu} S_{s}^{a c^{\prime}}(x)\right.\right. \\
& +S_{d}^{c b^{\prime}}(x) \gamma_{\nu} S_{s}^{\prime a a^{\prime}}(x) \gamma_{\mu} S_{u}^{b c^{\prime}}(x)+S_{s}^{c a^{\prime}}(x) \gamma_{\nu} S_{d}^{\prime b b^{\prime}}(x) \gamma_{\mu} S_{u}^{a c^{\prime}}(x) \\
& +S_{s}^{c b^{\prime}}(x) \gamma_{\nu} S_{u}^{\prime a a^{\prime}}(x) \gamma_{\mu} S_{d}^{b c^{\prime}}(x)+S_{u}^{c a^{\prime}}(x) \gamma_{\nu} S_{s}^{\prime b b^{\prime}}(x) \gamma_{\mu} S_{d}^{a c^{\prime}}(x) \\
& +S_{u}^{c b^{\prime}}(x) \gamma_{\nu} S_{d}^{a a^{\prime}}(x) \gamma_{\mu} S_{s}^{b c^{\prime}}(x)+S_{s}^{c c^{\prime}}(x) \operatorname{Tr}\left[S_{d}^{b a^{\prime}}(x) \gamma_{\nu} S_{u}^{\prime a b^{\prime}}(x) \gamma_{\mu}\right] \\
& \left.\left.+S_{u}^{c c^{\prime}}(x) \operatorname{Tr}\left[S_{s}^{b a^{\prime}}(x) \gamma_{\nu} S_{d}^{\prime a b^{\prime}}(x) \gamma_{\mu}\right]+S_{d}^{c c^{\prime}}(x) \operatorname{Tr}\left[S_{u}^{b a^{\prime}}(x) \gamma_{\nu} S_{s}^{\prime a b^{\prime}}(x) \gamma_{\mu}\right]\right\}\right), \\
& \Pi_{\mu \nu}^{O P E, \Xi^{*}}(p)=\frac{i}{3} \epsilon_{a b c} \epsilon_{a^{\prime} b^{\prime} c^{\prime}} \int d^{4} x e^{i p x}\left\langle\left\{ 2 S_{s}^{c a^{\prime}}(x) \gamma_{\nu} S_{s}^{\prime a b^{\prime}}(x) \gamma_{\mu} S_{u}^{b c^{\prime}}(x)\right.\right. \\
& -2 S_{s}^{c b^{\prime}}(x) \gamma_{\nu} S_{s}^{\prime a a^{\prime}}(x) \gamma_{\mu} S_{u}^{b c^{\prime}}(x)+4 S_{s}^{c b^{\prime}}(x) \gamma_{\nu} S_{u}^{\prime b a^{\prime}}(x) \gamma_{\mu} S_{s}^{a c^{\prime}}(x) \\
& +2 S_{u}^{c a^{\prime}}(x) \gamma_{\nu} S_{s}^{\prime a b^{\prime}}(x) \gamma_{\mu} S_{s}^{b c^{\prime}}(x)-2 S_{u}^{c a^{\prime}}(x) \gamma_{\nu} S_{s}^{\prime b b^{\prime}}(x) \gamma_{\mu} S_{s}^{a c^{\prime}}(x) \\
& -S_{u}^{c c^{\prime}}(x) \operatorname{Tr}\left[S_{s}^{b a^{\prime}}(x) \gamma_{\nu} S_{s}^{\prime a b^{\prime}}(x) \gamma_{\mu}\right]+S_{u}^{c c^{\prime}}(x) \operatorname{Tr}\left[S_{s}^{b b^{\prime}}(x) \gamma_{\nu} S_{s}^{\prime a a^{\prime}}(x) \gamma_{\mu}\right] \\
& \left.\left.-4 S_{s}^{c c^{\prime}}(x) \operatorname{Tr}\left[S_{u}^{b a^{\prime}}(x) \gamma_{\nu} S_{s}^{\prime a b^{\prime}}(x) \gamma_{\mu}\right]\right\}\right\rangle
\end{aligned}
$$

and

$$
\begin{aligned}
\Pi_{\mu \nu}^{O P E, \Omega^{-}}(p) & =\epsilon_{a b c} \epsilon_{a^{\prime} b^{\prime} c^{\prime}} \int d^{4} x e^{i p x}\left\langle\left\{ S_{s}^{c a^{\prime}}(x) \gamma_{\nu} S_{s}^{\prime a b^{\prime}}(x) \gamma_{\mu} S_{s}^{b c^{\prime}}(x)\right.\right. \\
& -S_{s}^{c a^{\prime}}(x) \gamma_{\nu} S_{s}^{\prime b b^{\prime}}(x) \gamma_{\mu} S_{s}^{a c^{\prime}}(x)-S_{s}^{c b^{\prime}}(x) \gamma_{\nu} S_{s}^{\prime a a^{\prime}}(x) \gamma_{\mu} S_{s}^{b c^{\prime}}(x) \\
& +S_{s}^{c b^{\prime}}(x) \gamma_{\nu} S_{s}^{\prime b a^{\prime}}(x) \gamma_{\mu} S_{s}^{a c^{\prime}}(x)-S_{s}^{c c^{\prime}}(x) \operatorname{Tr}\left[S_{s}^{b a^{\prime}}(x) \gamma_{\nu} S_{s}^{\prime a b^{\prime}}(x) \gamma_{\mu}\right] \\
& \left.\left.+S_{s}^{c c c^{\prime}}(x) \operatorname{Tr}\left[S_{s}^{b b^{\prime}}(x) \gamma_{\nu} S_{s}^{\prime a a^{\prime}}(x) \gamma_{\mu}\right]\right\}\right\rangle,
\end{aligned}
$$

where $S^{\prime}=C S^{T} C$. Here, $S_{u, d, s}$ denotes the light quark propagator and it is given at the nuclear medium in the fixed-point gauge as [1]

$$
\begin{aligned}
S_{q}^{a b}(x) & \equiv\left\langle\psi_{0}\left|T\left[q^{a}(x) \bar{q}^{b}(0)\right]\right| \psi_{0}\right\rangle_{\rho_{N}} \\
& =\frac{i}{2 \pi^{2}} \delta^{a b} \frac{1}{\left(x^{2}\right)^{2}} \not x-\frac{m_{q}}{4 \pi^{2}} \delta^{a b} \frac{1}{x^{2}}+\chi_{q}^{a}(x) \bar{\chi}_{q}^{b}(0) \\
& -\frac{i g_{s}}{32 \pi^{2}} F_{\mu \nu}^{A}(0) t^{a b, A} \frac{1}{x^{2}}\left[\not \sigma^{\mu \nu}+\sigma^{\mu \nu} \not x\right]+\ldots,
\end{aligned}
$$


where $\rho_{N}$ is the nuclear matter density, $m_{q}$ is the light quark mass, $\chi_{q}^{a}$ and $\bar{\chi}_{q}^{b}$ are the Grassmann background quark fields and $F_{\mu \nu}^{A}$ are classical background gluon fields. After inserting Eq. (23) in Eq. (19) - Eq. (22), we obtain the products of the Grassmann background quark fields and classical background gluon fields which correspond to the ground-state matrix elements of the corresponding quark and gluon operators [1]

$$
\begin{aligned}
\chi_{a \alpha}^{q}(x) \bar{\chi}_{b \beta}^{q}(0) & =\left\langle q_{a \alpha}(x) \bar{q}_{b \beta}(0)\right\rangle_{\rho_{N}}, \\
F_{\kappa \lambda}^{A} F_{\mu \nu}^{B} & =\left\langle G_{\kappa \lambda}^{A} G_{\mu \nu}^{B}\right\rangle_{\rho_{N}}, \\
\chi_{a \alpha}^{q} \bar{\chi}_{b \beta}^{q} F_{\mu \nu}^{A} & =\left\langle q_{a \alpha} \bar{q}_{b \beta} G_{\mu \nu}^{A}\right\rangle_{\rho_{N}}, \\
\text { and } & \\
\chi_{a \alpha}^{q} \bar{\chi}_{b \beta}^{q} \chi_{c \gamma}^{q} \bar{\chi}_{d \delta}^{q} & =\left\langle q_{a \alpha} \bar{q}_{b \beta} q_{c \gamma} \bar{q}_{d \delta}\right\rangle_{\rho_{N}} .
\end{aligned}
$$

Now, we need to define the quark, gluon and mixed condensates in nuclear matter. The matrix element $\left\langle q_{a \alpha}(x) \bar{q}_{b \beta}(0)\right\rangle_{\rho_{N}}$ is parameterized as [1]

$$
\begin{aligned}
\left\langle q_{a \alpha}(x) \bar{q}_{b \beta}(0)\right\rangle_{\rho_{N}}= & -\frac{\delta_{a b}}{12}\left[\left(\langle\bar{q} q\rangle_{\rho_{N}}+x^{\mu}\left\langle\bar{q} D_{\mu} q\right\rangle_{\rho_{N}}\right.\right. \\
+ & \left.\frac{1}{2} x^{\mu} x^{\nu}\left\langle\bar{q} D_{\mu} D_{\nu} q\right\rangle_{\rho_{N}}+\ldots\right) \delta_{\alpha \beta} \\
& +\left(\left\langle\bar{q} \gamma_{\lambda} q\right\rangle_{\rho_{N}}+x^{\mu}\left\langle\bar{q} \gamma_{\lambda} D_{\mu} q\right\rangle_{\rho_{N}}\right. \\
+ & \left.\left.\frac{1}{2} x^{\mu} x^{\nu}\left\langle\bar{q} \gamma_{\lambda} D_{\mu} D_{\nu} q\right\rangle_{\rho_{N}}+\ldots\right) \gamma_{\alpha \beta}^{\lambda}\right]
\end{aligned}
$$

The quark-gluon mixed condensate in nuclear matter is written as

$$
\begin{aligned}
& \left\langle g_{s} q_{a \alpha} \bar{q}_{b \beta} G_{\mu \nu}^{A}\right\rangle_{\rho_{N}} \\
& =-\frac{t_{a b}^{A}}{96}\left\{\left\langle g_{s} \bar{q} \sigma \cdot G q\right\rangle_{\rho_{N}}\left[\sigma_{\mu \nu}+i\left(u_{\mu} \gamma_{\nu}-u_{\nu} \gamma_{\mu}\right) \not\right]_{\alpha \beta}\right. \\
& +\left\langle g_{s} \bar{q} \not u \sigma \cdot G q\right\rangle_{\rho_{N}}\left[\sigma_{\mu \nu} \not u+i\left(u_{\mu} \gamma_{\nu}-u_{\nu} \gamma_{\mu}\right)\right]_{\alpha \beta} \\
& -4\left(\langle\bar{q} u \cdot D u \cdot D q\rangle_{\rho_{N}}+i m_{q}\langle\bar{q} \not u \cdot D q\rangle_{\rho_{N}}\right) \\
& \left.\times\left[\sigma_{\mu \nu}+2 i\left(u_{\mu} \gamma_{\nu}-u_{\nu} \gamma_{\mu}\right) \not u\right]_{\alpha \beta}\right\},
\end{aligned}
$$

where $t_{a b}^{A}$ are Gell-Mann matrices and $D_{\mu}=\frac{1}{2}\left(\gamma_{\mu} D+/\right.$ $D \gamma_{\mu}$ ). The matrix element of the four-dimension gluon condensate can also be parameterized as

$$
\begin{aligned}
\left\langle G_{\kappa \lambda}^{A} G_{\mu \nu}^{B}\right\rangle_{\rho_{N}} & =\frac{\delta^{A B}}{96}\left[\left\langle G^{2}\right\rangle_{\rho_{N}}\left(g_{\kappa \mu} g_{\lambda \nu}-g_{\kappa \nu} g_{\lambda \mu}\right)\right. \\
& \left.+O\left(\left\langle\mathbf{E}^{2}+\mathbf{B}^{2}\right\rangle_{\rho_{N}}\right)\right],
\end{aligned}
$$

where we ignore from the last term in this equation because of its negligible contribution. The different condensates in the above equations are defined in the following way $[1,28]$ :

$$
\begin{aligned}
\left\langle\bar{q} \gamma_{\mu} q\right\rangle_{\rho_{N}} & =\langle\bar{q} \not \mu q\rangle_{\rho_{N}} u_{\mu}, \\
\left\langle\bar{q} D_{\mu} q\right\rangle_{\rho_{N}} & =\langle\bar{q} u \cdot D q\rangle_{\rho_{N}} u_{\mu}=-i m_{q}\langle\bar{q} \not \mu q\rangle_{\rho_{N}} u_{\mu}, \\
\left\langle\bar{q} \gamma_{\mu} D_{\nu} q\right\rangle_{\rho_{N}}= & \frac{4}{3}\langle\bar{q} \not u \cdot D q\rangle_{\rho_{N}}\left(u_{\mu} u_{\nu}-\frac{1}{4} g_{\mu \nu}\right) \\
+ & \frac{i}{3} m_{q}\langle\bar{q} q\rangle_{\rho_{N}}\left(u_{\mu} u_{\nu}-g_{\mu \nu}\right), \\
\left\langle\bar{q} D_{\mu} D_{\nu} q\right\rangle_{\rho_{N}}= & \frac{4}{3}\langle\bar{q} u \cdot D u \cdot D q\rangle_{\rho_{N}}\left(u_{\mu} u_{\nu}-\frac{1}{4} g_{\mu \nu}\right) \\
& -\frac{1}{6}\left\langle g_{s} \bar{q} \sigma \cdot G q\right\rangle_{\rho_{N}}\left(u_{\mu} u_{\nu}-g_{\mu \nu}\right), \\
\left\langle\bar{q} \gamma_{\lambda} D_{\mu} D_{\nu} q\right\rangle_{\rho_{N}}= & 2\langle\bar{q} \not u \cdot D u \cdot D q\rangle_{\rho_{N}} \\
& {\left[u_{\lambda} u_{\mu} u_{\nu}-\frac{1}{6}\left(u_{\lambda} g_{\mu \nu}+u_{\mu} g_{\lambda \nu}+u_{\nu} g_{\lambda \mu}\right)\right] } \\
& -\frac{1}{6}\left\langle g_{s} \bar{q} \not u \sigma \cdot G q\right\rangle_{\rho_{N}}\left(u_{\lambda} u_{\mu} u_{\nu}-u_{\lambda} g_{\mu \nu}\right),
\end{aligned}
$$

where, in their derivations, the equation of motion has been used and the terms $O\left(m_{q}^{2}\right)$ have been neglected due to their ignorable contributions [11].

By substituting the above matrix elements and the inmedium condensates, after lengthy calculations, we find the expression of the correlation function in coordinate space. Using the relation,

$$
\begin{aligned}
\frac{1}{\left(x^{2}\right)^{n}}= & \int \frac{d^{D} t}{(2 \pi)^{D}} e^{-i t \cdot x} i(-1)^{n+1} 2^{D-2 n} \pi^{D / 2} \\
& \times \frac{\Gamma(D / 2-n)}{\Gamma(n)}\left(-\frac{1}{t^{2}}\right)^{D / 2-n}
\end{aligned}
$$

We transform the calculations to the momentum space. Then, by the help of the replacement

$$
\Gamma\left(\frac{D}{2}-n\right)\left(-\frac{1}{L}\right)^{\frac{D}{2}-n} \rightarrow \frac{(-1)^{n-1}}{(n-2) !}(-L)^{n-2} \ln (-L),
$$

we find the imaginary parts of the obtained results for different structures called the spectral densities $\Delta \rho_{i}\left(p_{0}, \vec{p}\right)$ in OPE side in terms of $\left(p^{2}\right)^{n}$. After ordering the Dirac matrices like the physical side, we set $p^{2}=p_{0}^{2}-|\vec{p}|^{2}$ and replace $p_{0}$ with $w$. In order to remove the contributions of the negative energy particles, we multiply the OPE side 
by the weight function $\left(w-\bar{E}_{p}\right) e^{-\frac{w^{2}}{M^{2}}}$ like the physical side and perform the integral

$$
\Pi_{i}\left(w_{0}, \vec{p}\right)=\int_{-w_{0}}^{w_{0}} d w \Delta \rho_{i}(w, \vec{p})\left(w-\bar{E}_{p}\right) e^{-\frac{w^{2}}{M^{2}}} .
$$

By carrying out the integration over $w$, one can find the $\Pi_{i}\left(w_{0}, \vec{p}\right)$ functions in Borel scheme. By using $w_{0}=\sqrt{s_{0}^{*}}$, with $s_{0}^{*}$ being the continuum threshold in nuclear matter, and making some variable changing, we find the final expressions of the $\Pi_{i}\left(s_{0}^{*}, M^{2}\right)$ functions. As an example, we present the functions $\Pi_{i}\left(s_{0}^{*}, M^{2}\right)$ for $\Sigma^{*}$ which are obtained as

$$
\Pi_{i}\left(s_{0}^{*}, M^{2}\right)=\Pi_{i}^{p e r t}\left(s_{0}^{*}, M^{2}\right)+\sum_{k=3}^{k=6} \Pi_{i}^{k}\left(s_{0}^{*}, M^{2}\right)
$$

where "pert" denotes the perturbative contributions and the upper indices 3, 4, 5 and 6 stand for the nonperturbative contributions. These functions are obtained as

$$
\begin{aligned}
\Pi_{1}^{\text {pert }}\left(s_{0}^{*}, M^{2}\right) & =\frac{1}{512 \pi^{4}}\left[3 \bar{E}_{p} M^{2} \sqrt{s_{0}^{*}}\left(m_{d}+m_{u}+m_{s}\right)\left(3 M^{2}-4 \vec{p}^{2}+2 s_{0}^{*}\right)\right] e^{-\frac{s_{0}^{*}}{M^{2}}} \\
& -\frac{1}{1024 \pi^{4}} \int_{0}^{s_{0}^{*}} d s \frac{3 \bar{E}_{p}\left(m_{d}+m_{u}+m_{s}\right)\left(3 M^{4}-4 M^{2} \vec{p}^{2}+4 \vec{p}^{4}\right)}{\sqrt{s}} e^{-\frac{s}{M^{2}}}, \\
\Pi_{2}^{\text {pert }}\left(s_{0}^{*}, M^{2}\right) & =\frac{1}{640 \pi^{4}}\left[\bar{E}_{p} M^{2} \sqrt{s_{0}^{*}}\left(3 M^{2}-4 \vec{p}^{2}+2 s_{0}^{*}\right)\right] e^{-\frac{s_{0}^{*}}{M^{2}}} \\
& -\frac{1}{1280 \pi^{4}} \int_{0}^{s_{0}^{*}} d s \frac{\bar{E}_{p}\left(3 M^{4}-4 M^{2} \vec{p}^{2}+4 \vec{p}^{4}\right)}{\sqrt{s}} e^{-\frac{s}{M^{2}}}, \\
\Pi_{3}^{\text {pert }}\left(s_{0}^{*}, M^{2}\right) & =0,
\end{aligned}
$$




$$
\begin{aligned}
& \Pi_{1}^{3}\left(s_{0}^{*}, M^{2}\right)=\frac{M^{2} \sqrt{s_{0}^{*}}}{24 \pi^{2}}\left[\left(3 m_{s}+3 m_{d}-4 m_{q}\right)\left\langle u^{\dagger} u\right\rangle_{\rho_{N}}+\left(3 m_{u}+3 m_{s}-4 m_{q}\right)\left\langle d^{\dagger} d\right\rangle_{\rho_{N}}+\left(3 m_{u}+3 m_{d}-4 m_{s}\right)\left\langle s^{\dagger} s\right\rangle_{\rho_{N}}\right. \\
& \left.-2 \bar{E}_{p}\left(\langle\bar{s} s\rangle_{\rho_{N}}+\langle\bar{u} u\rangle_{\rho_{N}}+\langle\bar{d} d\rangle_{\rho_{N}}\right)\right] e^{-\frac{s_{0}^{*}}{M^{2}}} \\
& +\frac{1}{144 \pi^{2}} \int_{0}^{s_{0}^{*}} d s \frac{1}{\sqrt{s}}\left[4 \bar{E}_{p}\left(\left\langle\bar{d} i D_{0} i D_{0} d\right\rangle_{\rho_{N}}+\left\langle\bar{u} i D_{0} i D_{0} u\right\rangle_{\rho_{N}}+\left\langle\bar{s} i D_{0} i D_{0} s\right\rangle_{\rho_{N}}\right)-4 \bar{E}_{p}\left(\left\langle\bar{d} g_{s} \sigma G d\right\rangle_{\rho_{N}}\right.\right. \\
& \left.+\left\langle\bar{u} g_{s} \sigma G u\right\rangle_{\rho_{N}}+\left\langle\bar{s} g_{s} \sigma G s\right\rangle_{\rho_{N}}\right)-12 \bar{E}_{p}\left(m_{u}+m_{s}\right)\left\langle d^{\dagger} i D_{0} d\right\rangle_{\rho_{N}}-12 \bar{E}_{p}\left(m_{d}+m_{s}\right)\left\langle u^{\dagger} i D_{0} u\right\rangle_{\rho_{N}} \\
& -12 \bar{E}_{p}\left(m_{d}+m_{u}\right)\left\langle s^{\dagger} i D_{0} s\right\rangle_{\rho_{N}}-6 \bar{E}_{p}\left(m_{q} m_{s}+m_{q} m_{u}-M^{2}+2 \vec{p}^{2}\right)\langle\bar{d} d\rangle_{\rho_{N}} \\
& -6 \bar{E}_{p}\left(m_{q} m_{s}+m_{q} m_{d}-M^{2}+2 \vec{p}^{2}\right)\langle\bar{u} u\rangle_{\rho_{N}}-6 \bar{E}_{p}\left(m_{q} m_{u}+m_{q} m_{d}-M^{2}+2 \vec{p}^{2}\right)\langle\bar{s} s\rangle_{\rho_{N}} \\
& \left.+\left(12 m_{q}-9 m_{s}-9 m_{u}\right)\left\langle d^{\dagger} d\right\rangle_{\rho_{N}}+\left(12 m_{q}-9 m_{s}-9 m_{d}\right)\left\langle u^{\dagger} u\right\rangle_{\rho_{N}}+\left(12 m_{q}-9 m_{d}-9 m_{u}\right)\left\langle s^{\dagger} s\right\rangle_{\rho_{N}}\right] e^{-\frac{s}{M^{2}}} \text {, } \\
& \Pi_{2}^{3}\left(s_{0}^{*}, M^{2}\right)=\frac{M^{2} \sqrt{s_{0}^{*}}}{36 \pi^{2}}\left(\left\langle u^{\dagger} u\right\rangle_{\rho_{N}}+\left\langle d^{\dagger} d\right\rangle_{\rho_{N}}+\left\langle s^{\dagger} s\right\rangle_{\rho_{N}}\right) \\
& +\frac{1}{216 \pi^{2}} \int_{0}^{s_{0}^{*}} d s \frac{1}{\sqrt{s}}\left[4 \bar{E}_{p}\left(\left\langle d^{\dagger} i D_{0} d\right\rangle_{\rho_{N}}+\left\langle u^{\dagger} i D_{0} u\right\rangle_{\rho_{N}}+\left\langle s^{\dagger} i D_{0} s\right\rangle_{\rho_{N}}\right)+\bar{E}_{p}\left(27 m_{s}+27 m_{u}-10 m_{q}\right)\langle\bar{d} d\rangle_{\rho_{N}}\right. \\
& +\bar{E}_{p}\left(27 m_{s}+27 m_{d}-10 m_{q}\right)\langle\bar{u} u\rangle_{\rho_{N}}+\bar{E}_{p}\left(27 m_{u}+27 m_{d}-10 m_{q}\right)\langle\bar{s} s\rangle_{\rho_{N}} \\
& \left.-3 M^{2}\left(\left\langle u^{\dagger} u\right\rangle_{\rho_{N}}+\left\langle d^{\dagger} d\right\rangle_{\rho_{N}}\left\langle+s^{\dagger} s\right\rangle_{\rho_{N}}\right)\right] e^{-\frac{s}{M^{2}}} \\
& \Pi_{3}^{3}\left(s_{0}^{*}, M^{2}\right)=\frac{M^{2} \sqrt{s_{0}^{*}}}{216 \pi^{2}}\left[-32\left(\left\langle u^{\dagger} i D_{0} u\right\rangle_{\rho_{N}}+\left\langle d^{\dagger} i D_{0} d\right\rangle_{\rho_{N}}+\left\langle s^{\dagger} i D_{0} s\right\rangle_{\rho_{N}}\right)-9 \bar{E}_{p}\left(\left\langle u^{\dagger} u\right\rangle_{\rho_{N}}+\left\langle d^{\dagger} d\right\rangle_{\rho_{N}}+\left\langle s^{\dagger} s\right\rangle_{\rho_{N}}\right)\right. \\
& \left.+8 m_{q}\left(\langle\bar{u} u\rangle_{\rho_{N}}+\langle\bar{d} d\rangle_{\rho_{N}}+\langle\bar{s} s\rangle_{\rho_{N}}\right)\right] \\
& +\frac{1}{432 \pi^{2}} \int_{0}^{s_{0}^{*}} d s \frac{1}{\sqrt{s}}\left[12 \bar{E}_{p}\left(\left\langle d^{\dagger} i D_{0} i D_{0} d\right\rangle_{\rho_{N}}+\left\langle u^{\dagger} i D_{0} i D_{0} u\right\rangle_{\rho_{N}}+\left\langle s^{\dagger} i D_{0} i D_{0} s\right\rangle_{\rho_{N}}\right)\right. \\
& +32 M^{2}\left(\left\langle d^{\dagger} i D_{0} d\right\rangle_{\rho_{N}}+\left\langle u^{\dagger} i D_{0} u\right\rangle_{\rho_{N}}+\left\langle s^{\dagger} i D_{0} s\right\rangle_{\rho_{N}}\right)-8 M^{2} m q\left(\langle\bar{d} d\rangle_{\rho_{N}}+\langle\bar{u} u\rangle_{\rho_{N}}+\langle\bar{s} s\rangle_{\rho_{N}}\right) \\
& -7 \bar{E}_{p}\left(\left\langle d^{\dagger} g_{s} \sigma G d\right\rangle_{\rho_{N}}+\left\langle u^{\dagger} g_{s} \sigma G u\right\rangle_{\rho_{N}}+\left\langle s^{\dagger} g_{s} \sigma G s\right\rangle_{\rho_{N}}\right)-\bar{E}_{p}\left(54 m_{q} m_{s}+54 m_{q} m_{u}-9 M^{2}+18 \vec{p}^{2}\right)\left\langle d^{\dagger} d\right\rangle_{\rho_{N}} \\
& \left.-\bar{E}_{p}\left(54 m_{q} m_{d}+54 m_{q} m_{s}-9 M^{2}+18 \vec{p}^{2}\right)\left\langle u^{\dagger} u\right\rangle_{\rho_{N}}-\bar{E}_{p}\left(54 m_{q} m_{d}+54 m_{q} m_{u}-9 M^{2}+18 \vec{p}^{2}\right)\left\langle s^{\dagger} s\right\rangle_{\rho_{N}}\right] e^{-\frac{s}{M^{2}}}
\end{aligned}
$$

$$
\begin{aligned}
\Pi_{1}^{4}\left(s_{0}^{*}, M^{2}\right) & =\frac{1}{128 \pi^{2}}\left\langle\frac{\alpha_{s} G^{2}}{\pi}\right\rangle_{\rho_{N}} \int_{0}^{s_{0}^{*}} d w \frac{\bar{E}_{p}\left(m_{d}+m_{u}+m_{s}\right)}{\sqrt{w}} e^{-\frac{s}{M^{2}}}, \\
\Pi_{2}^{4}\left(s_{0}^{*}, M^{2}\right) & =\frac{1}{576 \pi^{2}}\left\langle\frac{\alpha_{s} G^{2}}{\pi}\right\rangle_{\rho_{N}} \int_{0}^{s_{0}^{*}} d s \frac{\bar{E}_{p}}{\sqrt{s}} e^{-\frac{s}{M^{2}}}, \\
\Pi_{3}^{4}\left(s_{0}^{*}, M^{2}\right) & =0,
\end{aligned}
$$

$$
\begin{aligned}
\Pi_{1}^{5}\left(s_{0}^{*}, M^{2}\right) & =\frac{1}{48 \pi^{2}}\left[4 m_{q}\left\langle s^{\dagger} i D_{0} s\right\rangle_{\rho_{N}}+4 m_{q}\left\langle d^{\dagger} i D_{0} d\right\rangle_{\rho_{N}}+4 m_{q}\left\langle u^{\dagger} i D_{0} u\right\rangle_{\rho_{N}}-4\left\langle\bar{d} i D_{0} i D_{0} d\right\rangle_{\rho_{N}}-4\left\langle\bar{s} i D_{0} i D_{0} s\right\rangle_{\rho_{N}}\right. \\
& \left.-\left\langle\bar{u} i D_{0} i D_{0} u\right\rangle_{\rho_{N}}-\left\langle\bar{d} g_{s} \sigma G d\right\rangle_{\rho_{N}}-\left\langle\bar{s} g_{s} \sigma G s\right\rangle_{\rho_{N}}-\left\langle\bar{u} g_{s} \sigma G u\right\rangle_{\rho_{N}}\right] \int_{0}^{s_{0}^{*}} d s \frac{\bar{E}_{p}}{\sqrt{s}} e^{-\frac{s}{M^{2}}}
\end{aligned}
$$

$\Pi_{2}^{5}\left(s_{0}^{*}, M^{2}\right)=0$,

$$
\begin{aligned}
\Pi_{3}^{5}\left(s_{0}^{*}, M^{2}\right)=-\frac{1}{72 \pi^{2}}\left[\left\langle d^{\dagger} g_{s} \sigma G d\right\rangle_{\rho_{N}}+\left\langle u^{\dagger} g_{s} \sigma G u\right\rangle_{\rho_{N}}+\left\langle s^{\dagger} g_{s} \sigma G s\right\rangle_{\rho_{N}}\right] \int_{0}^{s_{0}^{*}} d s \frac{\bar{E}_{p}}{\sqrt{s}} e^{-\frac{s}{M^{2}}}, \\
\Pi_{1}^{6}\left(s_{0}^{*}, M^{2}\right)=0, \\
\Pi_{2}^{6}\left(s_{0}^{*}, M^{2}\right)=0, \\
\Pi_{3}^{6}\left(s_{0}^{*}, M^{2}\right)=0 .
\end{aligned}
$$

\section{Sum Rules for Physical Observables: Numerical Results}

Having obtained the hadronic and OPE sides of the correlation function, we match them to find QCD sum 
sidered decuplet baryons:

$$
\begin{aligned}
\lambda_{D}^{* 2} m_{D}^{*} e^{-\frac{E_{p}^{2}}{M^{2}}} & =\Pi_{1}\left(s_{0}^{*}, M^{2}\right), \\
\lambda_{D}^{* 2} e^{-\frac{E_{p}^{2}}{M^{2}}} & =\Pi_{2}\left(s_{0}^{*}, M^{2}\right), \\
\lambda_{D}^{* 2} \Sigma_{\nu} e^{-\frac{E_{p}^{2}}{M^{2}}} & =\Pi_{3}\left(s_{0}^{*}, M^{2}\right) .
\end{aligned}
$$

Now, we proceed to numerically analyze the above sum rules in $\Delta^{0}, \Sigma^{*}, \Xi^{*}$ and $\Omega^{-}$channels both in vacuum and nuclear medium. The sum rules contain numerous pa-

\begin{tabular}{|c|c|}
\hline Input parameters & Values \\
\hline $\overrightarrow{|\vec{p}|}$ & $270 \mathrm{MeV}[11]$ \\
\hline$m_{u} ; m_{d} ; m_{s}$ & $2.2_{-0.4}^{0.6} \mathrm{MeV} ; 4.7_{-0.4}^{0.5} \mathrm{MeV} ; 96_{-4}^{+8} \mathrm{MeV}[26]$ \\
\hline$\rho_{N}$ & $(0.11)^{3} \mathrm{GeV}^{3}[11,27,28]$ \\
\hline$\left\langle q^{\dagger} q\right\rangle_{\rho_{N}} ;\left\langle s^{\dagger} s\right\rangle_{\rho_{N}}$ & $\frac{3}{2} \rho_{N} ; 0[11,27-29]$ \\
\hline$\langle\bar{q} q\rangle_{0} ;\langle\bar{s} s\rangle_{0}$ & $(-0.241)^{3} \mathrm{GeV}^{3} ; 0.8\langle\bar{q} q\rangle_{0}[30]$ \\
\hline$m_{q}$ & $0.5\left(m_{u}+m_{d}\right)[11,27,28]$ \\
\hline$\sigma_{N}$ & $0.059 \mathrm{GeV}[31]$ \\
\hline$y$ & $0.04 \pm 0.02[32] ; 0.066 \pm 0.011 \pm 0.002[33] ; 0.02(13)(10)[34]$ \\
\hline$\langle\bar{q} q\rangle_{\rho_{N}} ;\langle\bar{s} s\rangle_{\rho_{N}}$ & $\langle\bar{q} q\rangle_{0}+\frac{\sigma_{N}}{2 m_{q}} \rho_{N} ;\langle\bar{s} s\rangle_{0}+y \frac{\sigma_{N}}{2 m_{q}} \rho_{N}[11,27-29,35]$ \\
\hline$\left\langle q^{\dagger} g_{s} \sigma G q\right\rangle_{\rho_{N}} ;\left\langle s^{\dagger} g_{s} \sigma G s\right\rangle_{\rho_{N}}$ & $-0.33 \mathrm{GeV}^{2} \rho_{N} ;-y 0.33 \mathrm{GeV}^{2} \rho_{N}[11,27-29,35]$ \\
\hline$\left\langle q^{\dagger} i D_{0} q\right\rangle_{\rho_{N}} ;\left\langle s^{\dagger} i D_{0} s\right\rangle_{\rho_{N}}$ & $0.18 \mathrm{GeV} \rho_{N} ; \frac{m_{s}\langle\bar{s} s\rangle_{\rho_{N}}}{4}+0.02 \mathrm{GeV} \rho_{N}[11,27-29,35]$ \\
\hline$\left\langle\bar{q} i D_{0} q\right\rangle_{\rho_{N}} ;\left\langle\bar{s} i D_{0} s\right\rangle_{\rho_{N}}$ & $\frac{3}{2} m_{q} \rho_{N} \simeq 0 ; 0[11, \underline{27}-29,35]$ \\
\hline$m_{0}^{2}$ & $0.8 \mathrm{GeV}^{2}[30]$ \\
\hline$\left\langle\bar{q} g_{s} \sigma G q\right\rangle_{0} ;\left\langle\bar{s} g_{s} \sigma G s\right\rangle_{0}$ & $m_{0}^{2}\langle\bar{q} q\rangle_{0} ; m_{0}^{2}\langle\bar{s} s\rangle_{0}$ \\
\hline$\left\langle\bar{q} g_{s} \sigma G q\right\rangle_{\rho_{N}} ;\left\langle\bar{s} g_{s} \sigma G s\right\rangle_{\rho_{N}}$ & $\left\langle\bar{q} g_{s} \sigma G q\right\rangle_{0}+3 G e V^{2} \rho_{N} ;\left\langle\bar{s} g_{s} \sigma G s\right\rangle_{0}+3 y G e V^{2} \rho_{N}[11,27-29,35$ \\
\hline$\left\langle\bar{q} i D_{0} i D_{0} q\right\rangle_{\rho_{N}} ;\left\langle\bar{s} i D_{0} i D_{0} s\right\rangle_{\rho_{N}}$ & $0.3 \mathrm{GeV}^{2} \rho_{N}-\frac{1}{8}\left\langle\bar{q} g_{s} \sigma G q\right\rangle_{\rho_{N}}$ \\
\hline$\left\langle q^{\dagger} i D_{0} i D_{0} q\right\rangle_{\rho_{N}} ;\left\langle s^{\dagger} i D_{0} i D_{0} s\right\rangle_{\rho_{N}}$ & $\begin{array}{l}0.3 y \mathrm{GeV}^{2} \rho_{N}-\frac{1}{8}\left\langle\bar{s} g_{s} \sigma G s\right\rangle_{\rho_{N}}[11,27-29, \underline{35} \\
0.031 \mathrm{GeV}^{2} \rho_{N}-\frac{1}{12}\left\langle q^{\dagger} g_{s} \sigma G q\right\rangle_{N} ;\end{array}$ \\
\hline GYTPN & $0.031 y G e V^{2} \rho_{N}-\frac{1}{12}\left\langle s^{\dagger} g_{s} \sigma G s\right\rangle_{\rho_{N}}[11,27-29,35]$ \\
\hline$\left\langle\frac{\alpha_{s}}{\pi} G^{2}\right\rangle_{0}$ & $(0.33 \pm 0.04)^{4} \mathrm{GeV}^{4}[30]$ \\
\hline$\underline{\left\langle\frac{\alpha_{s}}{\pi} G^{2}\right\rangle_{\rho_{N}}}$ & $\left\langle\frac{\alpha_{s}}{\pi} G^{2}\right\rangle_{0}-0.65 G e V \rho_{N}[11,27,28]$ \\
\hline
\end{tabular}
rameters, numerical values of which are collected in table III.

TABLE II: Numerical values of input parameters.

Besides the above input parameters, the QCD sum rules depend also on two auxiliary parameters that should be fixed: the Borel parameter $M^{2}$ and the continuum threshold $s_{0}^{*}$. The continuum threshold is not totally arbitrary and it is correlated with the energy of the first excited state with the same quantum numbers as the interpolating currents for decuplet baryons. According to the standard prescriptions, we take the interval $\left(m_{D}+0.4\right)^{2} \mathrm{GeV}^{2} \leq s_{0}^{*} \leq\left(m_{D}+0.6\right)^{2} \mathrm{GeV}^{2}$. The standard criteria in calculating the working window of the Borel parameter is that not only the contributions of the higher resonances and continuum should be adequately suppressed, but the contributions of the higher dimensional condensates should be small and the perturbative contributions should exceed the nonperturbative ones. These criteria lead to the following intervals:

$$
\begin{array}{ll}
1.1 \mathrm{GeV}^{2} \leq M^{2} \leq 1.4 \mathrm{GeV}^{2} & \text { for } \Delta^{0} \\
1.5 \mathrm{GeV}^{2} \leq M^{2} \leq 1.9 \mathrm{GeV}^{2} & \text { for } \Sigma^{* 0} \\
2.2 \mathrm{GeV}^{2} \leq M^{2} \leq 2.5 \mathrm{GeV}^{2} & \text { for } \Xi^{*} \\
2.6 \mathrm{GeV}^{2} \leq M^{2} \leq 3.0 \mathrm{GeV}^{2} & \text { for } \Omega^{-}
\end{array}
$$

Making use of the working windows of the auxiliary parameters and the values of other inputs, as examples, we plot the in-medium mass, $m_{\Delta}^{*}$, residue, $\lambda_{\Delta}^{*}$, and vector self energy, $\Sigma_{\Delta}^{\nu}$, of the $\Delta$ baryon as functions of $M^{2}$ at different fixed values of the threshold parameter $s_{0}$ and central values of other input parameters in figures 1-3. From these figures we see that the in-medium mass and residue as well as the vector self energy demonstrate good stability with respect to $M^{2}$ in its working region. It is also clear that the results very weakly depend on the threshold parameter $s_{0}$ in its working window. 


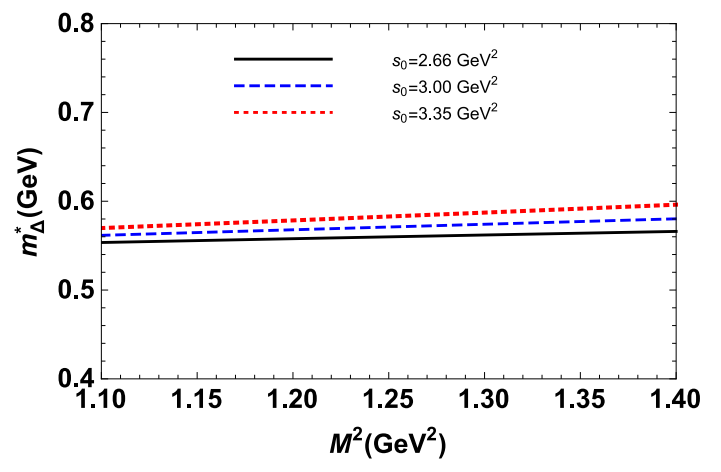

FIG. 1: The in-medium mass of the $\Delta$ baryon as a function of $M^{2}$ at different fixed values of the threshold parameter $s_{0}$ and central values of other input parameters.

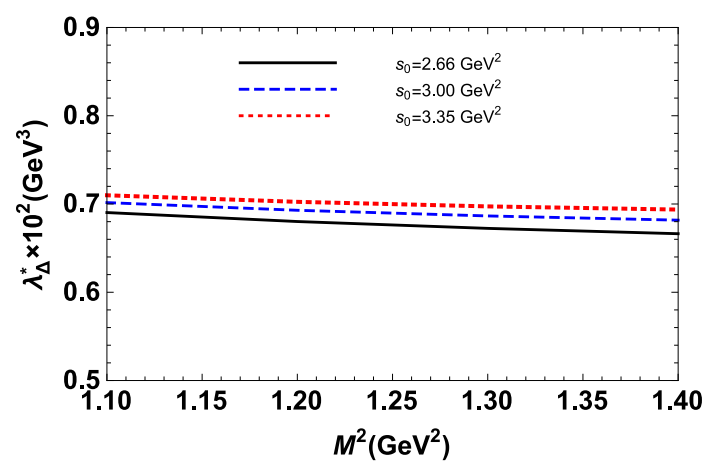

FIG. 2: The in-medium residue of the $\Delta$ baryon as a function of $M^{2}$ at different fixed values of the threshold parameter $s_{0}$ and central values of other input parameters.

In this part, we would like to briefly discuss the dependence of the results on the values of the three-momentum of the particles under consideration and the density of the nuclear matter. We work at zero temperature and, as is seen from table III, we take the external threemomentum of the quasi-particles approximately equal to Fermi momentum, $|\vec{p}|=270 \mathrm{MeV}$, in the numerical analysis. However, our numerical results show that

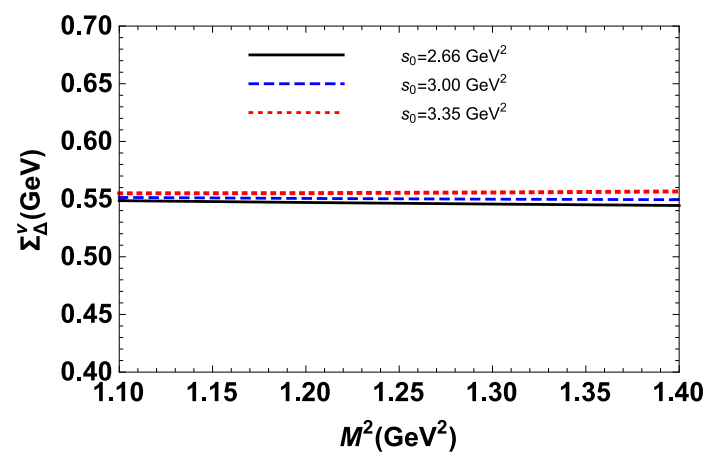

FIG. 3: The vector self energy of the $\Delta$ baryon as a function of $M^{2}$ at different fixed values of the threshold parameter $s_{0}$ and central values of other input parameters.

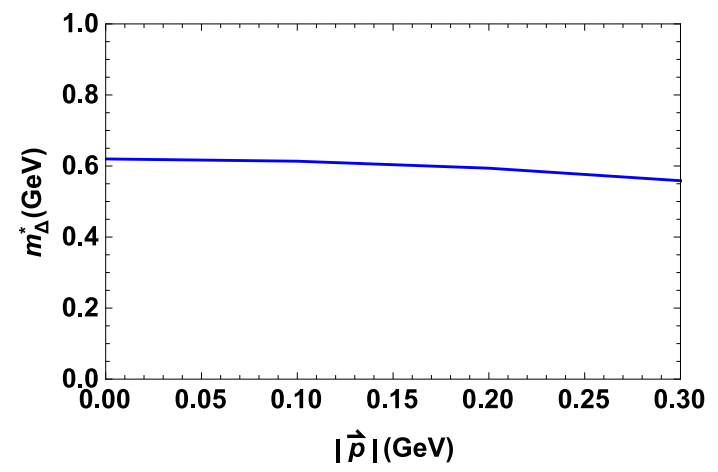

FIG. 4: The in-medium mass of the $\Delta$ baryon as a function of $|\vec{p}|$ at central values of all auxiliary and input parameters.

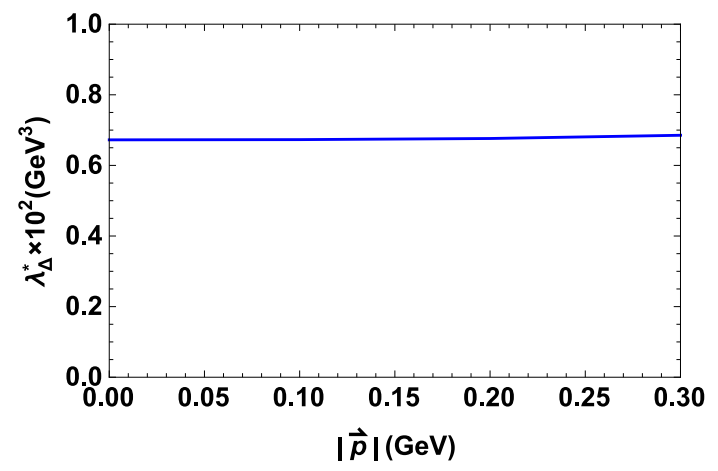

FIG. 5: The in-medium residue of the $\Delta$ baryon as a function of $|\vec{p}|$ at central values of all auxiliary and input parameters.

the physical quantities overall do not considerably depend on this parameter in the interval $[0,0.27] \mathrm{MeV}$ (see figures 4-6). This is an expected result. In the case of nucleons in nuclear matter, each quasi-nucleon has its own quasi-Fermi sea, hence, the external threemomentum of the quasi-nucleon is set at Fermi momentum at $\rho_{N}=0.16 \mathrm{fm}^{-3}=(110 \mathrm{MeV})^{3}[11,15]$. For a similar reason, the external three-momentum for the quasi-decuplet baryons, especially the strange members, can be easily set to zero. To see how the results behave

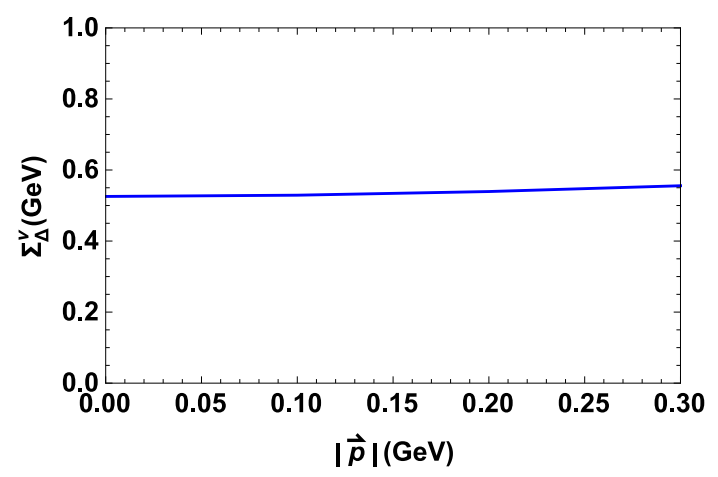

FIG. 6: The vector self-energy of the $\Delta$ baryon as a function of $|\vec{p}|$ at central values of all auxiliary and input parameters. 


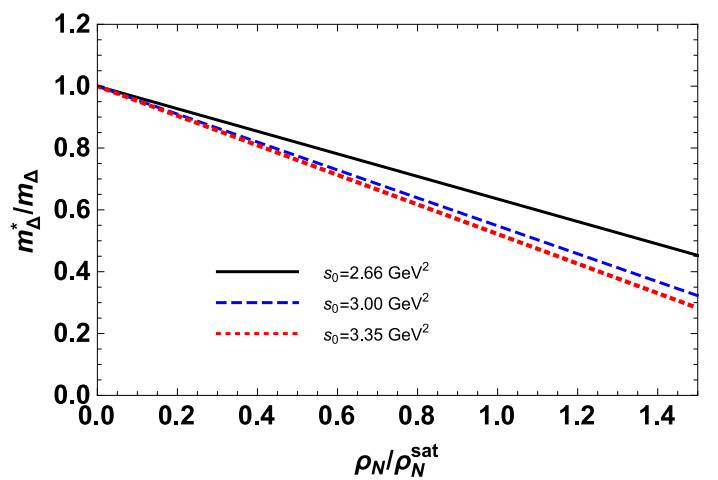

FIG. 7: $m_{\Delta}^{*} / m_{\Delta}$ versus $\rho_{N} / \rho_{N}^{s a t}$ at central values of $M^{2}$ and other input parameters.

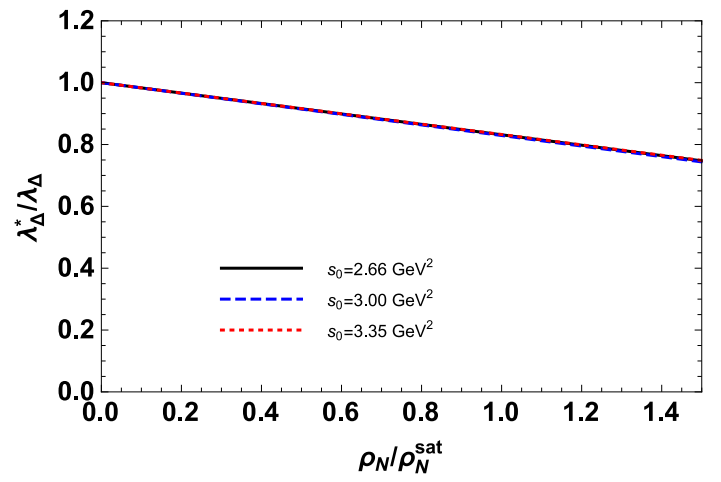

FIG. 8: $\lambda_{\Delta}^{*} / \lambda_{\Delta}$ versus $\rho_{N} / \rho_{N}^{s a t}$ at central values of $M^{2}$ and other input parameters.

with respect to the nuclear matter density, we show the dependence of the ratio of the mass and residue of, for instance, the $\Delta$ baryon in nuclear matter $\left(m_{\Delta}^{*}, \lambda_{\Delta}^{*}\right)$ to the mass and residue in vacuum $\left(m_{\Delta}, \lambda_{\Delta}\right)$ as well as $\Sigma_{\Delta}^{\nu} / m_{\Delta}^{*}$ on $\rho_{N} / \rho_{N}^{s a t}$, with $\rho_{N}^{s a t}=(0.11)^{3} \mathrm{GeV}^{3}$ being the saturation density used in the analysis, in figures (7-9). From these figures we see that the results depend linearly on the nuclear matter density.

After numerical analyses of the results for all baryons,

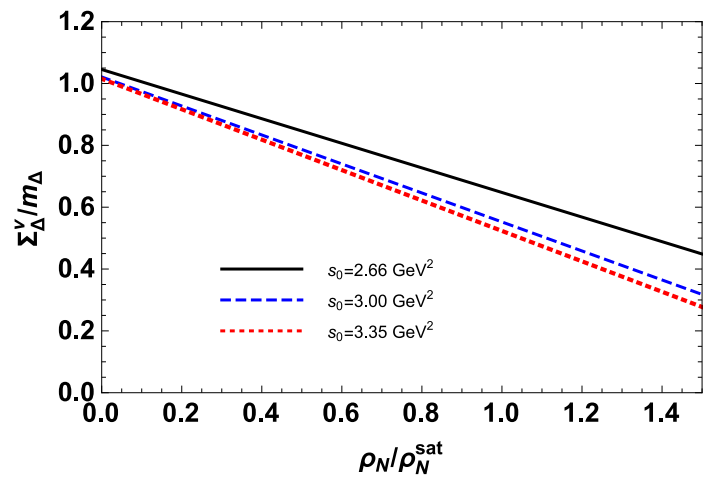

FIG. 9: $\Sigma_{\Delta}^{\nu} / m_{\Delta}$ versus $\rho_{N} / \rho_{N}^{\text {sat }}$ at central values of $M^{2}$ and other input parameters.

using the values presented in tableII, we find the values of the masses and residues both in nuclear matter and vacuum. We also obtain the vector and scalar self energies of the baryons under consideration in nuclear medium. Note that the vacuum results are obtained from those of the in-medium when $\rho_{N} \rightarrow 0$. The average values for the considered physical quantities are presented in table III. The errors quoted in this table correspond to the uncertainties in the calculations of the working regions for the auxiliary parameters as well as those coming from the errors of other input parameters.

\begin{tabular}{|c|c|c|c|c|c|c|}
\hline \hline & $\lambda_{\Delta}\left[\mathrm{GeV}^{3}\right]$ & $\lambda_{\Delta}^{*}\left[\mathrm{GeV}^{3}\right]$ & $m_{\Delta}[\mathrm{GeV}]$ & $m_{\Delta}^{*}[\mathrm{GeV}]$ & $\Sigma_{\Delta}^{\nu}[\mathrm{MeV}]$ & $\Sigma_{\Delta}^{S}[\mathrm{MeV}]$ \\
\hline Present study & $0.013 \pm 0.004$ & $0.007 \pm 0.002$ & $1.297 \pm 0.364$ & $0.571 \pm 0.159$ & $550 \pm 51$ & -726 \\
\hline \hline & $\lambda_{\Sigma^{*}}\left[\mathrm{GeV}^{3}\right]$ & $\lambda_{\Sigma^{*}}^{*}\left[\mathrm{GeV}^{3}\right]$ & $m_{\Sigma^{*}}[\mathrm{GeV}]$ & $m_{\Sigma^{*}}^{*}[\mathrm{GeV}]$ & $\Sigma_{\Sigma^{*}}^{\nu}[\mathrm{MeV}]$ & $\Sigma_{\Sigma^{*}}^{S}[\mathrm{MeV}]$ \\
\hline Present study & $0.024 \pm 0.007$ & $0.016 \pm 0.005$ & $1.385 \pm 0.387$ & $0.927 \pm 0.259$ & $409 \pm 41$ & -458 \\
\hline \hline & $\lambda_{\Xi^{*}}\left[\mathrm{GeV}^{3}\right]$ & $\lambda_{\Xi^{*}}^{*}\left[\mathrm{GeV}^{3}\right]$ & $m_{\Xi^{*}}[\mathrm{GeV}]$ & $m_{\Xi^{*}}^{*}[\mathrm{GeV}]$ & $\Sigma_{\Xi^{*}}^{\nu}[\mathrm{MeV}]$ & $\Sigma_{\Xi^{*}}^{S}[\mathrm{MeV}]$ \\
\hline Present study & $0.035 \pm 0.011$ & $0.027 \pm 0.008$ & $1.523 \pm 0.426$ & $1.399 \pm 0.392$ & $148 \pm 15$ & -124 \\
\hline \hline & $\lambda_{\Omega^{-}}\left[\mathrm{GeV}^{3}\right]$ & $\lambda_{\Omega^{-}}^{*}\left[\mathrm{GeV}^{3}\right]$ & $m_{\Omega^{-}}[\mathrm{GeV}]$ & $m_{\Omega^{-}}^{*}[\mathrm{GeV}]$ & $\Sigma_{\Omega^{-}}^{\nu}[\mathrm{MeV}]$ & $\Sigma_{\Omega^{-}}^{S}[\mathrm{MeV}]$ \\
\hline Present study & $0.044 \pm 0.013$ & $0.042 \pm 0.013$ & $1.668 \pm 0.467$ & $1.634 \pm 0.457$ & $46 \pm 5$ & -34 \\
\hline \hline
\end{tabular}

TABLE III: The numerical values of masses, residues and self-energies of $\Delta, \Sigma^{*}, \Xi^{*}$ and $\Omega^{-}$baryons.

From this table, first of all, we see that our predictions on the masses in vacuum are in good consistencies with the average experimental data presented in PDG [26]. The masses obtained in the nuclear medium show nega- tive shifts for all decuplet baryons. From the values of the scalar self energy $\left(\Sigma_{D}^{S}\right)$, demonstrating the shifts in the masses due to finite density, we deduce that the maximum shift in the masses, due to the nuclear medium, 
with amount of $56 \%$ belongs to the $\Delta$ baryon and its minimum, $2 \%$, corresponds to the $\Omega^{-}$state. This is an expected result since the $\Delta$ state have the same quark content as the nuclear medium and is more affected by the nuclear matter. When going from $\Delta$ to $\Omega^{-}$the up and down quarks are replaced with the strange quark. The $\Omega^{-}$state, having three $s$ quarks, is less affected by the medium. The small shifts in the parameters of $\Omega^{-}$ may be attributed to the intrinsic strangeness in the nucleons.

In the case of the residues, our predictions in vacuum are overall comparable with those obtained in [36, 37] within the errors. The small differences may be linked to different input parameters used in these works. The values of residues are also considerably affected by the medium. The shift in the residue of $\Delta$ with amount of $46 \%$ is maximum. The residue of $\Omega^{-}$again is minimally affected by the medium with amount of roughly $5 \%$.

The value of vector self energy is considerably large in all decuplet channels. It is again systematically reduced when going from the $\Delta$ to $\Omega^{-}$baryon. Our results may be confronted with the experimental data of
$\bar{P}$ ANDA Collaboration at FAIR and NICA facility. However, we should remark that those experiments correspond to heavy ion collisions and not exactly to a nuclear medium. Hence, the appropriate way to make such comparison would be to present sum rules at finite density but where the density is introduced through the baryonic chemical potential. This offers the possibility of exploring a wide range of densities. We worked with the nuclear matter density since the the in-medium condensates are available as functions of nuclear matter density not chemical potential and we extracted the zero-density (vacuum) sum rules, as a means of normalizing the finite density sum rules, to compare the results with the available experimental data and other theoretical predictions in vacuum.

\section{ACKNOWLEDGEMENTS}

K. A. thanks Doğus University for the financial support through the grant BAP 2015-16-D1-B04.
[1] P.B. Demorest, T. Pennucci, S. M. Ransom, M. S. E. Roberts, and J. W. T. Hessels, Nature 467 (2010) 1081.

[2] J. Antoniadis, et al., Science 340 (2013) 1233232.

[3] N. U. Bastian and D. Blaschke, "Towards a new quarknuclear matter EoS for applications in astrophysics and heavy-ion collisions," J. Phys. Conf. Ser. 668, no. 1, 012042 (2016) arXiv:1511.05881 [nucl-th]].

[4] E. S. Fraga, A. Kurkela and A. Vuorinen, "Interacting quark matter equation of state for compact stars," Astrophys. J. 781, no. 2, L25 (2014) arXiv:1311.5154 [nuclth]].

[5] K. Kim, H. K. Lee and J. Lee, "Compact Star Matter: EoS with New Scaling Law," arXiv:1607.03235 [nucl-th].

[6] M. Drews and W. Weise, "From asymmetric nuclear matter to neutron stars: a functional renormalization group study," Phys. Rev. C 91 (2015) no.3, 035802 doi:10.1103/PhysRevC.91.035802 arXiv:1412.7655 [nucl-th]].

[7] S. Weissenborn, D. Chatterjee and J. Schaffner-Bielich, "Hyperons and massive neutron stars: vector repulsion and SU(3) symmetry," Phys. Rev. C 85, no. 6, 065802 (2012) Erratum: [Phys. Rev. C 90, no. 1, 019904 (2014)] arXiv:1112.0234 [astro-ph.HE]].

[8] S. Weissenborn, D. Chatterjee and J. Schaffner-Bielich, "Hyperons and massive neutron stars: the role of hyperon potentials," Nucl. Phys. A 881, 62 (2012) arXiv:1111.6049 [astro-ph.HE]].

[9] A. Drago, A. Lavagno, G. Pagliara and D. Pigato, "Early appearance of isobars in neutron stars," Phys. Rev. C 90, no. 6, 065809 (2014) doi:10.1103/PhysRevC.90.065809 arXiv:1407.2843 [astro-ph.SR]].

[10] B. Singh et al. [PANDA Collaboration], "Study of doubly strange systems using stored antiprotons," Nucl. Phys. A 954, 323 (2016).

[11] T. D. Cohen, R. J. Furnstahl, D. K. Griegel and
X. m. Jin, "QCD sum rules and applications to nuclear physics," Prog. Part. Nucl. Phys. 35, 221 (1995) hep-ph/9503315.

[12] E. G. Drukarev and E. M. Levin, "The Qcd Sum Rules And Nuclear Matter," JETP Lett. 48, 338 (1988) [Pisma Zh. Eksp. Teor. Fiz. 48, 307 (1988)].

[13] T. Hatsuda, H. Hogaasen and M. Prakash, "QCD sum rules and the Okamoto-Nolen-Schiffer anomaly," Phys. Rev. Lett. 66, 2851 (1991) Erratum: [Phys. Rev. Lett. 69, 1290 (1992)].

[14] C. Adami and G. E. Brown, "Isospin breaking in nuclear physics: The Nolen-Schiffer effect," Z. Phys. A 340, 93 (1991).

[15] K. Azizi and N. Er, "Properties of nucleon in nuclear matter: once more," Eur. Phys. J. C 74, 2904 (2014) arXiv:1401.1680 [hep-ph]].

[16] K. Azizi, N. Er and H. Sundu, "Positive and negative parity hyperons in nuclear medium," Phys. Rev. D 92, no. 5, 054026 (2015) arXiv:1506.02183 [hep-ph]].

[17] X. m. Jin and R. J. Furnstahl, "QCD sum rules for Lambda hyperons in nuclear matter," Phys. Rev. C 49, 1190 (1994).

[18] X. M. Jin and M. Nielsen, "QCD sum rules for Sigma hyperons in nuclear matter," Phys. Rev. C 51, 347 (1995) hep-ph/9405331.

[19] M. J. Savage and M. B. Wise, "Hyperon masses in nuclear matter," Phys. Rev. D 53, 349 (1996) hep-ph/9507288.

[20] T. Miyatsu and K. Saito, "Hyperons in nuclear matter," arXiv:0903.1893 [nucl-th].

[21] S. R. Beane et al., "Hyperon-Nucleon Interactions and the Composition of Dense Nuclear Matter from Quantum Chromodynamics," Phys. Rev. Lett. 109, 172001 (2012) arXiv:1204.3606 [hep-lat]].

[22] S. M. Ouellette and R. Seki, "Selfenergy of decuplet baryons in nuclear matter," Phys. Lett. B 404, 108 
(1997) hep-ph/9702422.

[23] Y. Chung, H. G. Dosch, M. Kremer and D. Schall, "Baryon Sum Rules and Chiral Symmetry Breaking," Nucl. Phys. B 197, 55 (1982).

[24] R. Thomas, T. Hilger and B. Kampfer, "Four-quark condensates in nucleon QCD sum rules," Nucl. Phys. A 795, 19 (2007) arXiv:0704.3004 [hep-ph]].

[25] B. D. Serot and J. D. Walecka, "The Relativistic Nuclear Many Body Problem," Adv. Nucl. Phys. 16, 1 (1986).

[26] C. Patrignani et al. (Particle Data Group), Chinese Physics C, 40, 100001 (2016)

[27] T. D. Cohen, R. J. Furnstahl and D. K. Griegel, "Quark and gluon condensates in nuclear matter," Phys. Rev. C 45, 1881 (1992).

[28] X. m. Jin, T. D. Cohen, R. J. Furnstahl and D. K. Griegel, "QCD sum rules for nucleons in nuclear matter. 2.," Phys. Rev. C 47, 2882 (1993).

[29] Z. G. Wang, "Analysis of the doubly heavy baryons in the nuclear matter with the QCD sum rules," Eur. Phys. J. C 72, 2099 (2012) arXiv:1205.0605 [hep-ph]].

[30] V. M. Belyaev and B. L. Ioffe, "Determination of the baryon mass and baryon resonances from the quantumchromodynamics sum rule. Strange baryons," Sov. Phys. JETP 57, 716 (1983) [Zh. Eksp. Teor. Fiz. 84, 1236 (1983)]; B. L. Ioffe, "QCD at low energies," Prog. Part. Nucl. Phys. 56, 232 (2006) hep-ph/0502148.
[31] J. M. Alarcon, J. Martin Camalich and J. A. Oller, "The chiral representation of the $\pi N$ scattering amplitude and the pion-nucleon sigma term," Phys. Rev. D 85, 051503 (2012) arXiv:1110.3797 [hep-ph]].

[32] A. W. Thomas, P. E. Shanahan and R. D. Young, "Strangeness in the nucleon: what have we learned?," Nuovo Cim. C 035N04, 3 (2012) arXiv:1202.6407 [nuclth]].

[33] S. Dinter, V. Drach and K. Jansen, "Dark matter search and the scalar quark contents of the nucleon," Int. J. Mod. Phys. Proc. Suppl. E 20, 110 (2011) arXiv:1111.5426 [hep-lat]].

[34] J. M. Alarcon, L. S. Geng, J. Martin Camalich and J. A. Oller, "The strangeness content of the nucleon from effective field theory and phenomenology," Phys. Lett. B 730, 342 (2014) arXiv:1209.2870 [hep-ph]].

[35] X. m. Jin, M. Nielsen, T. D. Cohen, R. J. Furnstahl and D. K. Griegel, "QCD Sum rules for nucleons in nuclear matter. 3.," Phys. Rev. C 49, 464 (1994).

[36] F. X. Lee, "Predicative ability of QCD sum rules for decuplet baryons," Phys. Rev. C 57, 322 (1998) hep-ph/9707332.

[37] K. Azizi and G. Bozkir, "Decuplet baryons in a hot medium," Eur. Phys. J. C 76, no. 10, 521 (2016) arXiv:1606.05452 [hep-ph]]. 